\title{
Anomalous hydrodynamics in a class of scarred frustration-free Hamiltonians
}

\author{
Jonas Richter $\odot^{*}$ and Arijeet Pal $\odot$ \\ Department of Physics and Astronomy, University College London, Gower Street, London WC1E 6BT, United Kingdom
}

(Received 13 August 2021; accepted 9 December 2021; published 14 January 2022)

\begin{abstract}
Atypical eigenstates in the form of quantum scars and fragmentation of Hilbert space due to conservation laws provide obstructions to thermalization in the absence of disorder. In certain models with dipole and U(1) conservation, the fragmentation results in subdiffusive transport. In this Letter we study the interplay between scarring and weak fragmentation giving rise to anomalous hydrodynamics in a class of one-dimensional spin-1 frustration-free projector Hamiltonians, known as deformed Motzkin chain. The ground states and low-lying excitations of these chains exhibit large entanglement and critical slowdown. We show that at high energies the particular form of the projectors causes the emergence of disjoint Krylov subspaces for open boundary conditions, with an exact quantum scar being embedded in each subspace, leading to slow growth of entanglement and localized dynamics for specific out-of-equilibrium initial states. Furthermore, focusing on infinite temperature, we unveil that spin transport is subdiffusive, which we corroborate by simulations of constrained stochastic cellular automaton circuits. Compared with dipole moment conserving systems, the deformed Motzkin chain appears to belong to a different universality class with distinct dynamical transport exponent and only polynomially many Krylov subspaces.
\end{abstract}

DOI: 10.1103/PhysRevResearch.4.L012003

Introduction. Unraveling the intricate dynamics of isolated many-body quantum systems has attracted a vast amount of interest in recent years [1-5]. In this context, transport processes represent arguably one of the most generic nonequilibrium situations, and the common expectation is that hydrodynamics emerges naturally from the underlying unitary time evolution [6,7]. The emergence of a variety of universal hydrodynamics and their relevance to transport coefficients are actively pursued theoretically with potential for utility in near-term quantum devices [6-9]. Enormous experimental efforts have been undertaken to study quantum transport in various platforms, including mesoscopic and solid-state settings as well as cold-atom quantum simulators (see, e.g., Refs. [10-14]), remarkably allowing one to observe even anomalous types of hydrodynamics $[15,16]$.

While most quantum systems relax to thermal equilibrium, as explained by the eigenstate thermalization hypothesis (ETH) [17-19] and numerically confirmed for a variety of models (e.g., Refs. [3,20-28]), several counterexamples to this paradigm have been identified, with integrable and many-body localized systems being prime examples [29-31]. Moreover, studies of the so-called PXP model revealed that also weaker violations of the ETH are possible, where rare nonthermal states coexist with thermal eigenstates at the same energy density [32,33], now usually referred to as quantum

\footnotetext{
*j.richter@ucl.ac.uk

Published by the American Physical Society under the terms of the Creative Commons Attribution 4.0 International license. Further distribution of this work must maintain attribution to the author(s) and the published article's title, journal citation, and DOI.
}

many-body scars [33-39]. By now, quantum scars have been found in various models [33-53], and tailored embedding procedures further allow one to place nonthermal eigenstates into the spectrum of chaotic many-body Hamiltonians [54,55].

Building on insights from fractonic systems [56-58], the phenomenon of Hilbert-space fragmentation provides yet another mechanism to break ergodicity [59-62]. Hilbert-space fragmentation occurs, for instance, in locally interacting models which in addition to a $\mathrm{U}(1)$ charge also conserve the associated dipole moment, though other possibilities have been discussed as well [63-67]. In such cases, the Hilbert space splits into exponentially many disconnected blocks, often referred to as Krylov subspaces, despite states in different subspaces having the same symmetries. While some subspaces might be integrable or localized, others can be chaotic $[62,64,68]$. Even within the thermalizing regimes of such models, the constraints on excitations, e.g., higherorder conservation laws, have implications on the dynamics and lead to subdiffusive transport [69-76], reminiscent of disordered models close to the many-body localization transition [77-79]. The class of frustration-free Hamiltonians considered in this Letter similarly exhibits disjoint Krylov subspaces and subdiffusive hydrodynamics. The underlying mechanisms, however, will be distinct from those of the models mentioned above.

Another motivation for this Letter is given by recent work on quantum many-body scars and Hilbert-space fragmentation in Fredkin chains [80]. The Fredkin model is a spin- $1 / 2$ chain, where the Hamiltonian is a sum over projectors and can be rewritten in the form of a dressed Heisenberg chain [81], bearing resemblance to other kinetically constrained models [82-84]. While the model is nonintegrable in general, its degenerate ground-state manifold is known analytically [81]. 
(a)

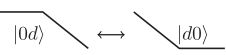

(b)

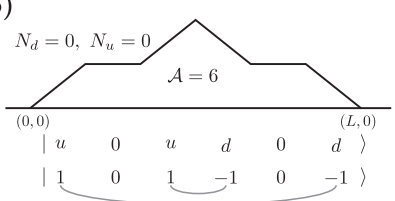

(c)

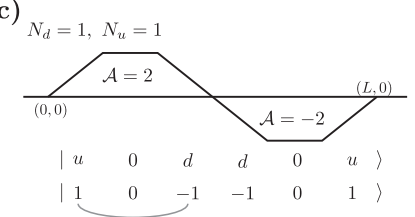

FIG. 1. Identification of $|1\rangle,|-1\rangle,|0\rangle$ as $|u\rangle,|d\rangle,|0\rangle$, corresponding to up, down, and horizontal moves on a plane. (a) Local updates induced by the projectors of $\mathcal{H}_{v}$. (b) and (c) For OBCs, the Hilbert space splits into Krylov subspaces labeled by $N_{d}$ and $N_{u}$. (b) shows an example configuration with $N_{d}=N_{u}=0$. Paired spins are indicated by arcs. (c) shows a configuration with $N_{d}=N_{u}=1$. The area $\mathcal{A}$ determines the weight of the basis state within $\left|S_{v}\right\rangle$; see Eq. (3).

In particular, as shown in Ref. [80], the degenerate states can be moved to the center of the spectrum by generalizing the model $[85,86]$, with each state belonging to a different Krylov subspace.

Here, we consider a closely related class of models, known as deformed Motzkin chain [87-93]. While the ground-state properties of Motzkin chains have been explored in a series of works [87-96], much less is known about the nature of thermalization and nonequilibrium dynamics. In this Letter, we show that the particular form of the Hamiltonian leads to an intriguing interplay of disconnected Krylov subspaces and exact quantum many-body scars, similar to Ref. [80]. As a main result, we unveil that the Motzkin chain exhibits subdiffusive hydrodynamics at infinite temperature, which we corroborate by simulations of suitable stochastic cellular automaton circuits [69,71,72,97,98]. Furthermore, we demonstrate that the scarred eigenstates lead to localized dynamics for specific out-of-equilibrium states and parameter regimes.

The model. We consider a class of spin-1 projector Hamiltonians, $\mathcal{H}_{v}=\sum_{\ell} \Pi_{\ell, \ell+1}(v)$, known as deformed Motzkin chain [87-96],

$$
\Pi_{\ell, \ell+1}(v)=c_{1}\left|D_{v}\right\rangle\left\langle D_{v}\left|+c_{2}\right| U_{v}\right\rangle\left\langle U_{v}\left|+c_{3}\right| V_{v}\right\rangle\left\langle V_{v}\right|,
$$

where $c_{1}, c_{2}$, and $c_{3}$ are real-valued coefficients, $v \geqslant$ 0 is a deformation parameter, and the terms $|\cdot\rangle\langle\cdot|$ are given by $|D\rangle=(|0 d\rangle-v|d 0\rangle) / \sqrt{1+v^{2}}, \quad|U\rangle=(|u 0\rangle-$ $v|0 u\rangle) / \sqrt{1+v^{2}},|V\rangle=(|u d\rangle-v|00\rangle) / \sqrt{1+v^{2}}$ and should be understood as acting on two neighboring sites $\ell$ and $\ell+1$. We adopt the convention to denote the three eigenstates of a local spin-1 operator $S_{\ell}^{z}$ as $|u\rangle \equiv|+1\rangle,|d\rangle \equiv|-1\rangle$ and $|0\rangle$, where $|u\rangle$ ("up"), $|d\rangle$ ("down"), and $|0\rangle$ are interpreted as the moves $(x, y) \rightarrow(x+1, y+1),(x, y) \rightarrow(x+1, y-1)$, and $(x, y) \rightarrow(x+1, y)$ on a two-dimensional plane [89]; see Fig. 1. The terms $|\cdot\rangle\langle\cdot|$ in Eq. (1) have eigenvalues 0 and 1 such that $\mathcal{H}_{\nu}$ has a positive-semidefinite spectrum if all $c_{i} \geqslant 0$. $\mathcal{H}_{v}$ has a U(1) symmetry, such that $S^{z}=\sum_{\ell} S_{\ell}^{z}$ is conserved. Written in terms of usual spin-1 operators, $\mathcal{H}_{v}$ takes on a bilinear-biquadratic form $[91,94,95]$.

For a spin configuration on $L$ sites, the identification of spins as moves leads to a "random walk." In the $S^{z}=0$ sector, these walks start at $(0,0)$ and end at $(L, 0)$; see Figs. 1(b) and 1(c). For open boundary conditions (OBCs), an important concept is then the distinction between paired and unpaired moves [87]. An up move is called unpaired if there is no matching down move further to the right in the chain, and a down move is unpaired if there is no matching up move further to the left. Given a configuration with no unpaired moves, the height profile never crosses the horizon [Fig. 1(b)]. Such walks in the upper half plane are referred to as Motzkin paths, giving rise to the name of the model.

Disconnected Krylov subspaces. In the case of OBCs, the Hilbert space of $\mathcal{H}_{v}$ splits into Krylov subspaces due to the interplay of the boundary conditions and the action of the projectors on neighboring spins; cf. Fig. 1(a). The subspaces can be understood as equivalence classes, where each spin configuration is equivalent to a specific root state $\left|\psi_{d u}\right\rangle$ [87]. Given an arbitrary configuration, $\left|\psi_{d u}\right\rangle$ can be defined as follows. First, identify pairs of up and down spins, where the spins forming a pair do not have to be nearest neighbors; cf. Figs. 1(b) and 1(c). Secondly, flip both spins to the $|0\rangle$ state and move the zeros to the center, which eventually yields [87]

$$
\left|\psi_{d u}\right\rangle=|\underbrace{d d \cdots d d}_{N_{d}} \underbrace{00 \cdots 00}_{L-N_{d}-N_{u}} \underbrace{u u \cdots u u}_{N_{u}}\rangle,
$$

where $N_{d}$ and $N_{u}$ denote the numbers of unpaired down or up moves. Given $\left|\psi_{d u}\right\rangle$, its corresponding Krylov subspace $\mathcal{K}_{d u}=\mathcal{K}\left(\mathcal{H}_{v},\left|\psi_{d u}\right\rangle\right)$ follows as $\mathcal{K}_{d u}=$ $\operatorname{span}\left\{\left|\psi_{d u}\right\rangle, \mathcal{H}_{v}\left|\psi_{d u}\right\rangle, \mathcal{H}_{v}^{2}\left|\psi_{d u}\right\rangle, \ldots\right\}$. In particular, two spin configurations which correspond to different $\left|\psi_{d u}\right\rangle$ cannot be transformed into each other by the action of $\mathcal{H}_{v}$. As an example, consider $\left|\psi_{1}\right\rangle=|u \cdots u d \cdots d\rangle$ and $\left|\psi_{2}\right\rangle=$ $|d \cdots d u \cdots u\rangle$, which both have $S^{z}=0$. However, while $\left|\psi_{1}\right\rangle$ belongs to $\mathcal{K}_{00}$ (i.e., it is equivalent to $|0 \cdots 0\rangle$ ), $\left|\psi_{2}\right\rangle$ belongs to $\mathcal{K}_{L / 2 L / 2}$. In fact, $\left|\psi_{2}\right\rangle$ is an exact eigenstate of $\mathcal{H}_{v}$, i.e., it spans a subspace of dimension one. Apparently, the degree of "Hilbert-space fragmentation" in the Motzkin chain is weaker compared with, e.g., models with charge and dipole conservation, which exhibit exponentially many subspaces [59-61]. For instance, in the $S^{z}=0$ sector, there are only $L / 2+1$ separate $\mathcal{K}_{d u}$ labeled by $0 \leqslant N_{d}=N_{u} \leqslant L / 2$, i.e., the total number of subspaces grows only polynomially with $L$. An expression for the dimension $\mathcal{D}_{d u}$ of each $\mathcal{K}_{d u}$ can be derived combinatorially [99]. In particular, for $\mathcal{K}_{d u}$ with small $N_{d}+N_{u}$, $\mathcal{D}_{d u}$ is expected to grow exponentially with $L$. At the same time, for any finite $L$, there always exist $\mathcal{K}_{d u}$ with $\mathcal{D}_{d u}=1$ (namely, when $N_{d}+N_{u}=L$ ), as well as small subspaces with $\mathcal{D}_{d u} \propto L$.

For subspaces with large $\mathcal{D}_{d u}$, thermalization is expected to occur. This is visualized in Fig. 2(a) in terms of the eigenstate entanglement entropy $S_{|n\rangle}=-\operatorname{Tr}\left[\rho_{A} \ln \rho_{A}\right]$, where $\rho_{A}=$ $\operatorname{Tr}_{B}\{|n\rangle\langle n|\}$ is the reduced density matrix for a half-chain bipartition. While the overall distribution of $S_{|n\rangle}$ is rather broad, it looks thermal when focusing on individual $\mathcal{K}_{d u}$ with small $N_{d}, N_{u}$. At the same time, the low values of $S_{|n\rangle}$ in the center of the spectrum mostly belong to $\mathcal{K}_{d u}$ with large $N_{d}, N_{u}$, where the maximally achievable entanglement is limited due to small $\mathcal{D}_{d u}$. Moreover, as shown in the Supplemental Material [100], individual $\mathcal{K}_{d u}$ indeed exhibit chaotic energy-level statistics, and most eigenstates follow the ETH.

Exact quantum many-body scars. Despite $\mathcal{H}_{v}$ being nonintegrable and chaotic, a number of eigenstates $\left|\mathcal{S}_{\nu}\right\rangle$ can be 

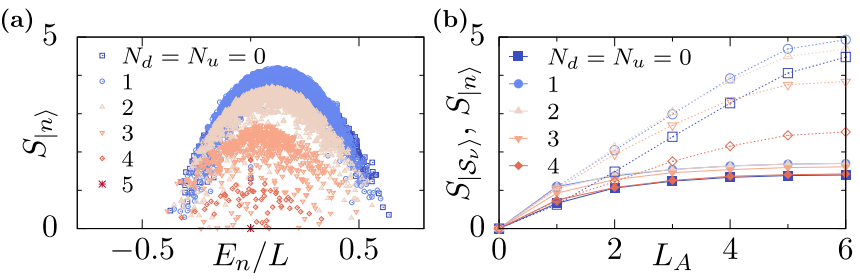

FIG. 2. (a) Eigenstate entanglement $S_{|n\rangle}$ for OBCs, $L=10$, and $v=1$, labeled according to their Krylov subspace. (b) $S_{\left|\mathcal{S}_{v}\right\rangle}$ (filled symbols with solid curves) at $v=1$ vs subsystem size $L_{A}$ for $L=12$ and different $\mathcal{K}_{d u}$ with $N_{d}=N_{u}$. As a comparison, the entanglement $S_{|n\rangle}$ (open symbols with dashed curves) of an eigenstate directly adjacent to $\left|\mathcal{S}_{v}\right\rangle$ is shown. We have $c_{1}=c_{3}=1$ and $c_{2}=-1$.

constructed combinatorially [87-89]. In this context, the key quantity is the area $\mathcal{A}_{k}$ enclosed by the height profile of a given spin configuration $|k\rangle$, where areas below the horizon contribute negatively; cf. Figs. 1(b) and 1(c). Within each $\mathcal{K}_{d u}$, $\left|\mathcal{S}_{\nu}\right\rangle$ is then given by the area-weighted superposition [87-89] (see also Supplemental Material [100])

$$
\left|\mathcal{S}_{\nu}\right\rangle=\frac{1}{\sqrt{M_{v}^{\prime}}} \sum_{k=1}^{\mathcal{D}_{d u}} v^{\mathcal{A}_{k}}|k\rangle=\frac{1}{\sqrt{M_{v}}} \sum_{k=1}^{\mathcal{D}_{d u}} v^{-\mathcal{P}_{k}}|k\rangle,
$$

where the sum runs over all $\mathcal{D}_{d u}$ basis states $|k\rangle, \mathcal{P}=$ $\sum_{\ell=1}^{L} \ell S_{\ell}^{z}$ is the dipole operator with $\mathcal{P}_{k}=\langle k|\mathcal{P}| k\rangle$, and $M_{v}^{\prime}$ and $M_{v}$ ensure normalization. The states $\left|\mathcal{S}_{v}\right\rangle$ have exactly zero energy as they are annihilated by all projectors in Eq. (1) [87-89]. According to Eq. (3), $\left|\mathcal{S}_{\nu}\right\rangle$ is dominated by $|k\rangle$ with large positive $\mathcal{P}_{k}$ if $v<1$. In contrast, for $v>1,|k\rangle$ with large negative $\mathcal{P}_{k}$ dominate. At $v=1,\left|\mathcal{S}_{v}\right\rangle$ is an equal-weight superposition of all states in $\mathcal{K}_{d u}$, reminiscent of the RokhsarKivelson ground state in quantum dimer models [107].

By choosing suitable $c_{i}$ in Eq. (1), the $\left|\mathcal{S}_{v}\right\rangle$ can be shifted close to the center of the spectrum [108], where they act as quantum many-body scars due to their subvolume-law entanglement [87-89], similar to other examples of frustration-free ground states being embedded by deforming the underlying model [43-45]. The nonthermal nature of the $\left|\mathcal{S}_{v}\right\rangle$ is emphasized in Fig. 2(b), where $S_{\left|\mathcal{S}_{\nu}\right\rangle}$ is shown versus subsystem size $L_{A}$ for different $\mathcal{K}_{d u}$. In particular, $S_{\left|\mathcal{S}_{v}\right\rangle}$ is compared with the entanglement of an eigenstate directly adjacent to $\left|\mathcal{S}_{\nu}\right\rangle$, demonstrating that typical eigenstates are extensively entangled whereas $\left|\mathcal{S}_{\nu}\right\rangle$ is not. As shown in the Supplemental Material [100], $\left|\mathcal{S}_{v}\right\rangle$ also violates the ETH by yielding atypical expectation values for local operators.

While the construction of $\mathcal{K}_{d u}$ as in Eq. (2) does not apply to periodic boundary conditions (PBCs), we note that quantum scars appear to exist also for PBCs [100].

Anomalous hydrodynamics. We probe the transport properties of $\mathcal{H}_{v}$ in terms of the infinite-temperature correlation function $C(r, t)$,

$$
C(r, t)=\operatorname{Tr}\left[S_{\ell+r}^{z}(t) S_{\ell}^{z}\right] / 3^{L},
$$

where $S_{\ell+r}^{z}(t)=e^{i \mathcal{H} t} S_{\ell+r}^{z} e^{-i \mathcal{H} t}$ and $r$ is the distance between the two sites [109]. In the case of diffusion, $C(r, t)$ takes on a Gaussian shape with a standard deviation $\sigma(t) \propto t^{1 / z}$ with $z=2[6,110]$. Correspondingly, the autocorrelation function $C(r=0, t)$ acquires a hydrodynamic tail, $C(0, t) \propto t^{-1 / z}$. For
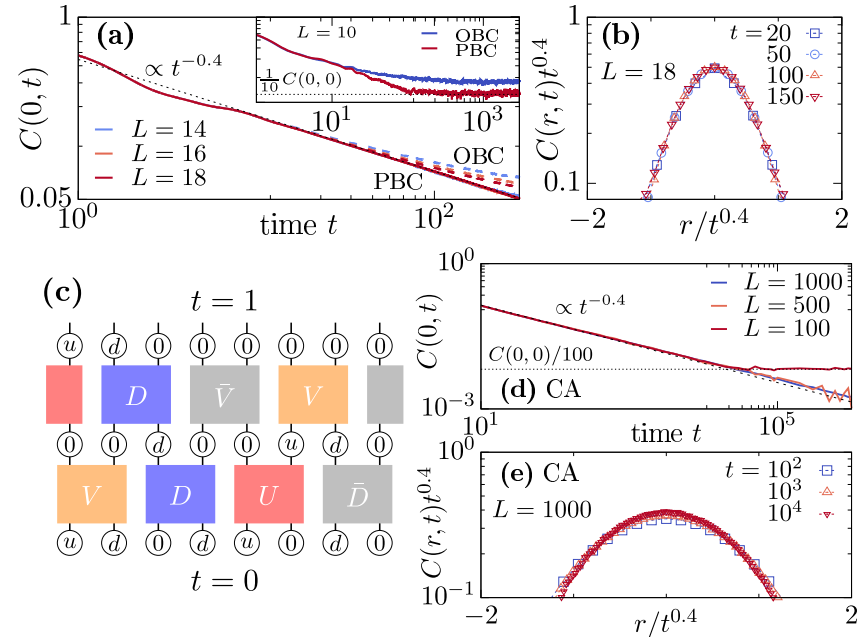

FIG. 3. (a) $C(0, t)$ for PBCs (solid curves) and OBCs (dashed curves) at $v=1$ and $L=14,16,18$. A power law $\propto t^{-1 / z}$ with $z=5 / 2$ is shown for comparison (thin dashed line). The inset shows data for $L=10$ up to longer times. (b) $C(r, t) t^{1 / z}$ vs $r / t^{1 / z}$ at fixed times. We have $c_{1}=c_{3}=1$ and $c_{2}=-1$ in all cases. (c) Exemplary time step in the cellular automaton (CA) circuit, consisting of two layers of two-site updates. Given a particular configuration of two sites, one of the updates $D, U$, or $V$ is chosen, while with probability $1 / 2$, we instead apply $\bar{D}, \bar{U}$, or $\bar{V}$, leaving the spin configuration unchanged (see Supplemental Material [100] for more details). (d) and (e) Analogous data to those in (a) and (b), but now obtained by CA circuits for larger $L$.

a thermalizing system, one expects a uniform distribution at long times, $C(r, t \rightarrow \infty) \rightarrow C_{\text {eq }}$, where $C_{\text {eq }}=C(0,0) / L[6]$. We exploit quantum typicality $[100,111,112]$ to simulate $C(r, t)$ for spin-1 systems up to $L=18$, beyond the range of full exact diagonalization. Focusing on $v=1$, we find that $C(0, t) \propto t^{-1 / z}$ with $z \approx 5 / 2$ (similar to Ref. [76]), suggesting that spin transport in the Motzkin chain is not diffusive but subdiffusive instead, both for PBCs and OBCs [Fig. 3(a)]. In the latter case, the power law persists on a shorter time scale as $C(0, t)$ saturates to a higher long-time value $C(0, t \rightarrow \infty)>$ $C_{\text {eq }}$ [inset of Fig. 3(a)] due to the disjoint $\mathcal{K}_{d u}$. We expect this difference between PBCs and OBCs to disappear in the thermodynamic limit $L \rightarrow \infty$, where the exponentially large $\mathcal{K}_{d u}$ dominate. Subdiffusive spin transport is further substantiated in Fig. 3(b), where the correlations $C(r, t)$ for different $t$ nicely collapse onto each other if the data and $r$ are rescaled with $t^{1 / z}$. We note that the observed value of $z$ is distinct from that found in dipole-conserving systems, where $z=4[60,71]$.

Intuitively, the occurrence of subdiffusion can be understood by considering the updates of local spin configurations induced by $\mathcal{H}_{v}$; cf. Fig. 1(a). As there are no matrix elements connecting $|d u\rangle \leftrightarrow|00\rangle$ or $|d u\rangle \leftrightarrow|u d\rangle$, configurations $|d u\rangle$ act as bottlenecks. Particularly, extended regions of the form $\mid \cdots d d$ dиии... will slow down the dynamics. This argument can also be stated more formally by inspecting the spin-current operator of $\mathcal{H}_{v}$; see Supplemental Material [100]. While we cannot provide a full hydrodynamic theory, we here proceed by constructing a stochastic cellular automaton (CA) circuit (see Fig. 3(c) and Supplemental Material [100]) which mimics the terms appearing in $\mathcal{H}_{v}$ and allows us to 


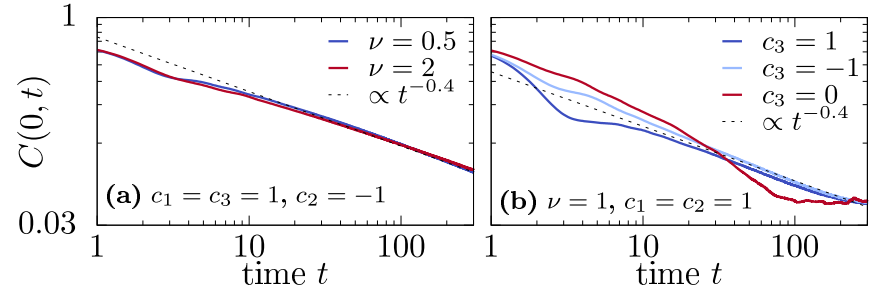

FIG. 4. (a) $C(0, t)$ at $v=0.5,2$ for $c_{1}=c_{3}=1$ and $c_{2}=-1$. A power law $\propto t^{-0.4}$ is shown for comparison. (b) $C(0, t)$ at $v=1$ for $c_{3}=-1,0,1$ and $c_{1}=c_{2}=1$. We have $L=16$ and PBCs in all cases.

access large systems and long times $[69,71,72,97,98]$. The soobtained data for $L \leqslant 10^{3}$ and $t \leqslant 10^{6}$ in Figs. 3(d) and 3(e) corroborate our findings of anomalous hydrodynamics with $z \approx 5 / 2$ at infinite temperature. (Our CA data for large $L$ and long $t$ are also consistent with $z \approx 8 / 3$ [113].) Putting these results into perspective, we note that subdiffusive dynamics in Motzkin chains [92,94,95] (and related Fredkin models [114]) has been observed before at low temperatures by analyzing the scaling of low-lying energy gaps, where a slightly larger $z$ was found. In this context, we note that the dynamical exponent $z$ in certain constrained chaotic models consisting of Floquet random unitary circuits can be related to the scaling of the low-energy gap of Rokhsar-Kivelson-type Hamiltonians using classical Markov circuits [75,113,115,116], which has partially motivated our usage of CA circuits.

While we have focused on $v=1$ in Fig. 3, we stress that the occurrence of high-temperature subdiffusion seems robust for a wider range of parameters. This is demonstrated in Fig. 4, where $C(0, t) \propto t^{-1 / z}$ both for $v=0.5,2$ and for $v=1$ but different choices of $c_{i}$. Only for $c_{3}=0$ does the decay of $C(0, t)$ appear to be different, which can be explained by the fact that $\mathcal{H}_{\nu}$ becomes integrable in this limit [117].

As an aside, we note that the anomalous transport properties of $\mathcal{H}_{v}$ also reflect themselves in an unusual growth of Rényi entropies $S_{\alpha}(t)=\ln \operatorname{Tr}\left[\rho_{A}^{\alpha}\right] /(1-\alpha), \rho_{A}=$ $\operatorname{Tr}_{B}|\psi(t)\rangle\langle\psi(t)|$, which were argued to grow sub-ballistically for $\alpha>1[118,119]$; see Supplemental Material [100] for details.

Initial-state dependence. While $C(r, t)$ represents a hightemperature average, studying quantum quenches with individual out-of-equilibrium states reveals the impact of the quantum scar $\left|\mathcal{S}_{v}\right\rangle$ on the dynamics. In particular, given its construction in Eq. (3), the dynamics can be tuned between different regimes depending on the deformation parameter $v$. We here exemplify this fact by considering a domain wall $|\psi\rangle=|u \cdots u d \cdots d\rangle$, which is a natural initial condition for quench dynamics [120-123]. While $|\psi\rangle$ has zero energy density, $\left\langle\psi\left|\mathcal{H}_{v}\right| \psi\right\rangle / L \rightarrow 0$, such that thermalization is expected, we note that in the picture of random walks on a plane (Fig. 1), $|\psi\rangle$ maximizes the area $\mathcal{A}$. According to the construction of $\left|\mathcal{S}_{\nu}\right\rangle$ in Eq. (3), $|\psi\rangle$ therefore contributes dominantly to $\left|\mathcal{S}_{v}\right\rangle$ if $v>1$ (here, $\left|\left\langle\psi \mid \mathcal{S}_{v}\right\rangle\right|^{2} \approx 0.64$ for $v=2$ and $L=16$ [124], in contrast to $\left|\left\langle\psi \mid \mathcal{S}_{v}\right\rangle\right|^{2}=1 / \mathcal{D}_{d u}^{2}$ for $\left.v=1\right)$. As a consequence, we find that $\mathcal{L}(t)=|\langle\psi(t) \mid \psi\rangle|^{2}$ decays quickly for $v=0.5,1$, while $\mathcal{L}(t)$ oscillates around a finite value for $v=2$ [Fig. 5(a)]. Likewise, the growth of the von Neumann entropy $S_{1}(t)$ [126] is significantly slower for $v=2$
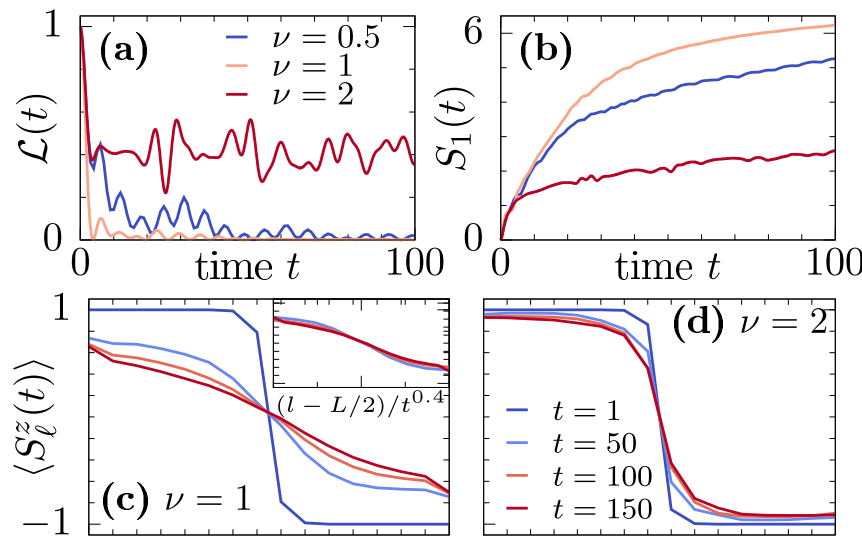

1

site $\ell$

161

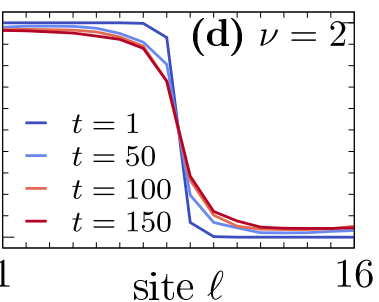

FIG. 5. Dynamics of domain-wall state for $L=16$ and OBCs. (a) and (b) $\mathcal{L}(t)=|\langle\psi(t) \mid \psi\rangle|^{2}$ and $S_{1}(t)$ for $v=0.5,1,2$. (c) and (d) $\left\langle S_{\ell}^{z}(t)\right\rangle$ at fixed $t$ for $v=1,2$. The inset in (c) shows data at $t=$ $50,100,150$ vs $(\ell-L / 2) / t^{0.4}$.

[Fig. 5(b)]. As shown in the Supplemental Material [100], there also exist initial states where dynamics is instead slower for $v<1$ and faster for $v>1$.

By tuning $v$ and thereby controlling its overlap with $\left|\mathcal{S}_{v}\right\rangle$, it is thus possible to obstruct thermalization of $|\psi\rangle$. This is emphasized even more in Figs. 5(c) and 5(d), where the spin profiles $\left\langle S_{\ell}(t)\right\rangle=\left\langle\psi(t)\left|S_{\ell}^{z}\right| \psi(t)\right\rangle$ are shown at fixed times for $v=1$ and $v=2$. In particular, for $v=2,\left\langle S_{\ell}(t)\right\rangle$ is found to remain localized even at long times. In contrast, for $v=1$, $|\psi\rangle$ is not dominated by $\left|\mathcal{S}_{\nu}\right\rangle$ such that the domain wall melts away, albeit $\left\langle S_{\ell}(t)\right\rangle$ is still rather inhomogeneous even at $t=$ 150. In fact, the profiles for different $t$ approximately collapse onto a single curve when plotted against $(l-L / 2) / t^{1 / z}$ [inset of Fig. 5(c)], i.e., consistent with the anomalous transport discussed above. We note that similar parameter-dependent melting of domain-wall states is known for other classes of models as well [120,123].

Conclusion and outlook. To summarize, we have studied a class of frustration-free Hamiltonians, where disjoint Krylov subspaces, anomalous hydrodynamics, and exact quantum many-body scars occur simultaneously. Compared with dipole-conserving or other fractonic models, the Motzkin chain appears to lie in a different "universality class" featuring a distinct dynamical transport exponent $z \approx 5 / 2$ at infinite temperature and Hilbert-space fragmentation with only polynomially many subspaces. The quantum scars $\left|\mathcal{S}_{v}\right\rangle$ are similar to other embeddings of frustration-free ground states by deforming the underlying model [43-45]. Moreover, a similar construction of exact scars in individual Krylov subspaces has been recently presented for related Fredkin chains [80].

Regarding prospective directions of research, we note that while at present an analytical expression is known only for the states $\left|\mathcal{S}_{v}\right\rangle$, the data in Fig. 2 suggest that $\mathcal{H}_{v}$ hosts other low-entangled eigenstates beyond $\left|\mathcal{S}_{v}\right\rangle$. Approximating further nonthermal eigenstates, e.g., by devising a spectrum generating algebra [127-130] acting on $\left|\mathcal{S}_{\nu}\right\rangle$, might thus be an interesting attempt. Another extension is to study hydrodynamics at finite temperatures to connect our hightemperature results to the subdiffusive scaling of low-energy 
excitations [94,95], as well as to consider transport beyond half filling, where CA circuits have already proven helpful [72]. Finally, the stability of the $\left|\mathcal{S}_{\nu}\right\rangle$ and, particularly, the persistence of anomalous hydrodynamics upon adding different perturbations to $\mathcal{H}_{v}$ are open questions.
We thank S. Moudgalya, B. Ware, and R. Vasseur for helpful comments. This work was funded by the European Research Council (ERC) under the European Union's Horizon 2020 research and innovation program (Grant Agreement No. 853368).
[1] A. Polkovnikov, K. Sengupta, A. Silva, and M. Vengalattore, Colloquium: Nonequilibrium dynamics of closed interacting quantum systems, Rev. Mod. Phys. 83, 863 (2011).

[2] C. Gogolin and J. Eisert, Equilibration, thermalisation, and the emergence of statistical mechanics in closed quantum systems, Rep. Prog. Phys. 79, 056001 (2016).

[3] L. D’Alessio, Y. Kafri, A. Polkovnikov, and M. Rigol, From quantum chaos and eigenstate thermalization to statistical mechanics and thermodynamics, Adv. Phys. 65, 239 (2016).

[4] F. Borgonovi, F. M. Izrailev, L. F. Santos, and V. G. Zelevinsky, Quantum chaos and thermalization in isolated systems of interacting particles, Phys. Rep. 626, 1 (2016).

[5] T. Mori, T. N. Ikeda, E. Kaminshi, and M. Ueda, Thermalization and prethermalization in isolated quantum systems: a theoretical overview, J. Phys. B: At. Mol. Opt. Phys. 51, 112001 (2018).

[6] B. Bertini, F. Heidrich-Meisner, C. Karrasch, T. Prosen, R. Steinigeweg, and M. Žnidarič, Finite-temperature transport in one-dimensional quantum lattice models, Rev. Mod. Phys. 93, 025003 (2021).

[7] V. Khemani, A. Vishwanath, and D. A. Huse, Operator Spreading and the Emergence of Dissipative Hydrodynamics under Unitary Evolution with Conservation Laws, Phys. Rev. X 8, 031057 (2018).

[8] B. Ye, F. Machado, C. D. White, R. S. K. Mong, and N. Y. Yao, Emergent Hydrodynamics in Nonequilibrium Quantum Systems, Phys. Rev. Lett. 125, 030601 (2020).

[9] J. Richter and A. Pal, Simulating Hydrodynamics on Noisy Intermediate-Scale Quantum Devices with Random Circuits, Phys. Rev. Lett. 126, 230501 (2021).

[10] S. Das Sarma, S. Adam, E. H. Hwang, and E. Rossi, Electronic transport in two-dimensional graphene, Rev. Mod. Phys. 83, 407 (2011).

[11] C. Hess, Heat transport of cuprate-based low-dimensional quantum magnets with strong exchange coupling, Phys. Rep. 811, 1 (2019).

[12] A. Scheie, N. E. Sherman, M. Dupont, S. E. Nagler, M. B. Stone, G. E. Granroth, J. E. Moore and D. A. Tennant, Detection of Kardar-Parisi-Zhang hydrodynamics in a quantum Heisenberg spin-1/2 chain, Nat. Phys. 17, 726 (2021).

[13] S. Hild, T. Fukuhara, P. Schauß, J. Zeiher, M. Knap, E. Demler, I. Bloch, and C. Gross, Far-from-Equilibrium Spin Transport in Heisenberg Quantum Magnets, Phys. Rev. Lett. 113, 147205 (2014).

[14] N. Jepsen, J. Amato-Grill, I. Dimitrova, W. W. Ho, E. Demler, and W. Ketterle, Spin transport in a tunable Heisenberg model realized with ultracold atoms, Nature (London) 588, 403 (2020).

[15] D. Wei, A. Rubio-Abadal, B. Ye, F. Machado, J. Kemp, K. Srakaew, S. Hollerith, J. Rui, S. Gopalakrishnan, N. Y. Yao, I. Bloch, and J. Zeiher, Quantum gas microscopy of KardarParisi-Zhang superdiffusion, arXiv:2107.00038.
[16] M. K. Joshi, F. Kranzl, A. Schuckert, I. Lovas, C. Maier, R. Blatt, M. Knap, and C. F. Roos, Observing emergent hydrodynamics in a long-range quantum magnet, arXiv:2107.00033.

[17] J. M. Deutsch, Quantum statistical mechanics in a closed system, Phys. Rev. A 43, 2046 (1991).

[18] M. Srednicki, Chaos and quantum thermalization, Phys. Rev. E 50, 888 (1994).

[19] M. Rigol, V. Dunjko, and M. Olshanii, Thermalization and its mechanism for generic isolated quantum systems, Nature (London) 452, 854 (2008).

[20] R. Steinigeweg, J. Herbrych, and P. Prelovšek, Eigenstate thermalization within isolated spin-chain systems, Phys. Rev. E 87, 012118 (2013).

[21] W. Beugeling, R. Moessner, and M. Haque, Finite-size scaling of eigenstate thermalization, Phys. Rev. E 89, 042112 (2014).

[22] H. Kim, T. N. Ikeda, and D. A. Huse, Testing whether all eigenstates obey the eigenstate thermalization hypothesis, Phys. Rev. E 90, 052105 (2014).

[23] E. J. Torres-Herrera and L. F. Santos, Local quenches with global effects in interacting quantum systems, Phys. Rev. E 89, 062110 (2014).

[24] R. Mondaini, K. R. Fratus, M. Srednicki, and M. Rigol, Eigenstate thermalization in the two-dimensional transverse field Ising model, Phys. Rev. E 93, 032104 (2016).

[25] D. Jansen, J. Stolpp, L. Vidmar, and F. Heidrich-Meisner, Eigenstate thermalization and quantum chaos in the Holstein polaron model, Phys. Rev. B 99, 155130 (2019).

[26] T. LeBlond, K. Mallayya, L. Vidmar, and M. Rigol, Entanglement and matrix elements of observables in interacting integrable systems, Phys. Rev. E 100, 062134 (2019).

[27] M. Brenes, T. LeBlond, J. Goold, and M. Rigol, Eigenstate Thermalization in a Locally Perturbed Integrable System, Phys. Rev. Lett. 125, 070605 (2020).

[28] J. Richter, A. Dymarsky, R. Steinigeweg, and J. Gemmer, Eigenstate thermalization hypothesis beyond standard indicators: Emergence of random-matrix behavior at small frequencies, Phys. Rev. E 102, 042127 (2020).

[29] F. H. L. Essler and M. Fagotti, Quench dynamics and relaxation in isolated integrable quantum spin chains, J. Stat. Mech.: Theory Exp. (2016) 064002.

[30] R. Nandkishore and D. A. Huse, Many-body localization and thermalization in quantum statistical mechanics, Annu. Rev. Condens. Matter Phys. 6, 15 (2015).

[31] D. A. Abanin, E. Altman, I. Bloch, and M. Serbyn, Colloquium: Many-body localization, thermalization, and entanglement, Rev. Mod. Phys. 91, 021001 (2019).

[32] H. Bernien, S. Schwartz, A. Keesling, H. Levine, A. Omran, H. Pichler, S. Choi, A. S. Zibrov, M. Endres, M. Greiner, V. Vuletić, and M. D. Lukin, Probing many-body dynamics on a 51-atom quantum simulator, Nature (London) 551, 579 (2017). 
[33] C. J. Turner, A. A. Michailidis, D. A. Abanin, M. Serbyn, and Z. Papić, Weak ergodicity breaking from quantum many-body scars, Nat. Phys. 14, 745 (2018).

[34] S. Moudgalya, S. Rachel, B. A. Bernevig, and N. Regnault, Exact excited states of nonintegrable models, Phys. Rev. B 98, 235155 (2018).

[35] S. Moudgalya, N. Regnault, and B. A. Bernevig, Entanglement of exact excited states of Affleck-Kennedy-Lieb-Tasaki models: Exact results, many-body scars, and violation of the strong eigenstate thermalization hypothesis, Phys. Rev. B 98, 235156 (2018).

[36] V. Khemani, C. R. Laumann, and A. Chandran, Signatures of integrability in the dynamics of Rydberg-blockaded chains, Phys. Rev. B 99, 161101(R) (2019).

[37] S. Choi, C. J. Turner, H. Pichler, W. W. Ho, A. A. Michailidis, Z. Papić, M. Serbyn, M. D. Lukin, and D. A. Abanin, Emergent SU(2) Dynamics and Perfect Quantum Many-Body Scars, Phys. Rev. Lett. 122, 220603 (2019).

[38] C.-J. Lin and O. I. Motrunich, Exact Quantum Many-Body Scar States in the Rydberg-Blockaded Atom Chain, Phys. Rev. Lett. 122, 173401 (2019).

[39] M. Serbyn, D. A. Abanin, and Z. Papić, Quantum many-body scars and weak breaking of ergodicity, Nat. Phys. 17, 675 (2021).

[40] M. Schecter and T. Iadecola, Weak Ergodicity Breaking and Quantum Many-Body Scars in Spin-1 XY Magnets, Phys. Rev. Lett. 123, 147201 (2019).

[41] T. Iadecola and M. Žnidarić, Exact Localized and Ballistic Eigenstates in Disordered Chaotic Spin Ladders and the Fermi-Hubbard Model, Phys. Rev. Lett. 123, 036403 (2019).

[42] A. J. A. James, R. M. Konik, and N. J. Robinson, Nonthermal States Arising from Confinement in One and Two Dimensions, Phys. Rev. Lett. 122, 130603 (2019).

[43] S. Ok, K. Choo, C. Mudry, C. Castelnovo, C. Chamon, and T. Neupert, Topological many-body scar states in dimensions one, two, and three, Phys. Rev. Research 1, 033144 (2019).

[44] J. Wildeboer, A. Seidel, N. S. Srivatsa, A. E. B. Nielsen, and O. Erten, Topological quantum many-body scars in quantum dimer models on the kagome lattice, Phys. Rev. B 104, L121103 (2021).

[45] K. Lee, R. Melendrez, A. Pal, and H. J. Changlani, Exact threecolored quantum scars from geometric frustration, Phys. Rev. B 101, 241111(R) (2020).

[46] P. A. McClarty, M. Haque, A. Sen, and J. Richter, Disorderfree localization and many-body quantum scars from magnetic frustration, Phys. Rev. B 102, 224303 (2020).

[47] Y. Kuno, T. Mizoguchi, and Y. Hatsugal, Flat band quantum scar, Phys. Rev. B 102, 241115(R) (2020).

[48] F. M. Surace, P. P. Mazza, G. Giudici, A. Lerose, A. Gambassi, and M. Dalmonte, Lattice Gauge Theories and String Dynamics in Rydberg Atom Quantum Simulators, Phys. Rev. X 10, 021041 (2020).

[49] H. Zhao, J. Vovrosh, F. Mintert, and J. Knolle, Quantum ManyBody Scars in Optical Lattices, Phys. Rev. Lett. 124, 160604 (2020).

[50] B. van Voorden, J. Minář, and K. Schoutens, Quantum manybody scars in transverse field Ising ladders and beyond, Phys. Rev. B 101, 220305(R) (2020).

[51] S. Pilatowsky-Cameo, D. Villaseñor, M. A. BastarracheaMagnani, S. Lerma-Hernández, L. F. Santos, and J. G. Hirsch,
Ubiquitous quantum scarring does not prevent ergodicity, Nat. Commun. 12, 852 (2021).

[52] J. Jeyaretnam, J. Richter, and A. Pal, Quantum scars and bulk coherence in a symmetry-protected topological phase, Phys. Rev. B 104, 014424 (2021).

[53] D. Banerjee and A. Sen, Quantum Scars from Zero Modes in an Abelian Lattice Gauge Theory on Ladders, Phys. Rev. Lett. 126, 220601 (2021).

[54] N. Shiraishi and T. Mori, Systematic Construction of Counterexamples to the Eigenstate Thermalization Hypothesis, Phys. Rev. Lett. 119, 030601 (2017).

[55] N. Shiraishi, Connection between quantum-many-body scars and the Affleck-Kennedy-Lieb-Tasaki model from the viewpoint of embedded Hamiltonians, J. Stat. Mech.: Theory Exp. (2019) 083103.

[56] C. Chamon, Quantum Glassiness in Strongly Correlated Clean Systems: An Example of Topological Overprotection, Phys. Rev. Lett. 94, 040402 (2005).

[57] M. Pretko, The fracton gauge principle, Phys. Rev. B 98, 115134 (2018).

[58] R. M. Nandkishore and M. Hermele, Fractons, Annu. Rev. Condens. Matter Phys. 10, 295 (2019).

[59] S. Pai, M. Pretko, and R. M. Nandkishore, Localization in Fractonic Random Circuits, Phys. Rev. X 9, 021003 (2019).

[60] P. Sala, T. Rakovszky, R. Verresen, M. Knap, and F. Pollmann, Ergodicity Breaking Arising from Hilbert Space Fragmentation in Dipole-Conserving Hamiltonians, Phys. Rev. X 10, 011047 (2020).

[61] V. Khemani, M. Hermele, and R. Nandkishore, Localization from Hilbert space shattering: From theory to physical realizations, Phys. Rev. B 101, 174204 (2020).

[62] S. Moudgalya, A. Prem, R. Nandkishore, N. Regnault, and B. A. Bernevig, Thermalization and its absence within Krylov subspaces of a constrained Hamiltonian, in Memorial Volume for Shoucheng Zhang (World Scientific, Singapore, 2021), Chap. 7, pp. 147-209.

[63] G. De Tomasi, D. Hetterich, P. Sala, and F. Pollmann, Dynamics of strongly interacting systems: From Fock-space fragmentation to many-body localization, Phys. Rev. B 100, 214313 (2019).

[64] Z.-C. Yang, F. Liu, A. V. Gorshkov, and T. Iadecola, HilbertSpace Fragmentation from Strict Confinement, Phys. Rev. Lett. 124, 207602 (2020).

[65] W.-H. Li, X. Deng, and L. Santos, Hilbert Space Shattering and Disorder-Free Localization in Polar Lattice Gases, Phys. Rev. Lett. 127, 260601 (2021).

[66] K. Lee, A. Pal, and H. J. Changlani, Frustration-induced emergent Hilbert space fragmentation, Phys. Rev. B 103, 235133 (2021).

[67] D. Hahn, P. A. McClarty, and D. J. Luitz, Information dynamics in a model with Hilbert space fragmentation, SciPost Phys. 11, 074 (2021).

[68] L. Herviou, J. H. Bardarson, and N. Regnault, Many-body localization in a fragmented Hilbert space, Phys. Rev. B 103, 134207 (2021).

[69] J. Iaconis, S. Vijay, and R. Nandkishore, Anomalous subdiffusion from subsystem symmetries, Phys. Rev. B 100, 214301 (2019).

[70] A. Gromov, A. Lucas, and R. M. Nandkishore, Fracton hydrodynamics, Phys. Rev. Research 2, 033124 (2020). 
[71] J. Feldmeier, P. Sala, G. De Tomasi, F. Pollmann, and M. Knap, Anomalous Diffusion in Dipole- and Higher-MomentConserving Systems, Phys. Rev. Lett. 125, 245303 (2020).

[72] A. Morningstar, V. Khemani, and D. A. Huse, Kinetically constrained freezing transition in a dipole-conserving system, Phys. Rev. B 101, 214205 (2020).

[73] P. Zhang, Subdiffusion in strongly tilted lattice systems, Phys. Rev. Research 2, 033129 (2020).

[74] J. Iaconis, A. Lucas, and R. Nandkishore, Multipole conservation laws and subdiffusion in any dimension, Phys. Rev. E 103, 022142 (2021).

[75] S. Moudgalya, A. Prem, D. A. Huse, and A. Chan, Spectral statistics in constrained many-body quantum chaotic systems, Phys. Rev. Research 3, 023176 (2021).

[76] P. Glorioso, J. Guo, F. Rodriguez-Nieva, and A. Lucas, Breakdown of hydrodynamics below four dimensions in a fracton fluid, arXiv:2105.13365.

[77] Y. Bar Lev, G. Cohen, and D. R. Reichmann, Absence of Diffusion in an Interacting System of Spinless Fermions on a One-Dimensional Disordered Lattice, Phys. Rev. Lett. 114, 100601 (2015).

[78] K. Agarwal, S. Gopalakrishnan, M. Knap, M. Müller, and E. Demler, Anomalous Diffusion and Griffiths Effects Near the Many-Body Localization Transition, Phys. Rev. Lett. 114, 160401 (2015).

[79] D. J. Luitz and Y. Bar Lev, The ergodic side of the manybody localization transition, Ann. Phys. (Berlin) 529, 1600350 (2017).

[80] C. Langlett and S. Xu, Hilbert space fragmentation and exact scars of generalized Fredkin spin chains, Phys. Rev. B 103, L220304 (2021).

[81] O. Salberger and V. Korepin, Entangled spin chain, Rev. Math. Phys. 29, 1750031 (2017).

[82] F. Ritort and P. Sollich, Glassy dynamics of kinetically constrained models, Adv. Phys. 52, 219 (2003).

[83] Z. Lan, M. van Horssen, S. Powell, and J. P. Garrahan, Quantum Slow Relaxation and Metastability due to Dynamical Constraints, Phys. Rev. Lett. 121, 040603 (2018).

[84] N. Pancotti, G. Giudice, J. I. Cirac, J. P. Garrahan, and M. C. Bañuls, Quantum East Model: Localization, Nonthermal Eigenstates, and Slow Dynamics, Phys. Rev. X 10, 021051 (2020).

[85] O. Salberger, T. Udagawa, Z. Zhang, H. Katsura, I. Klisch, and V. Korepin, Deformed Fredkin spin chain with extensive entanglement, J. Stat. Mech.: Theory Exp. (2017) 063103.

[86] K. Adhikari and K. S. D. Beach, Tunable quantum spin chain with three-body interactions, Phys. Rev. B 102, 184415 (2020).

[87] S. Bravyi, L. Caha, R. Movassagh, D. Nagaj, and P. W. Shor, Criticality without Frustration for Quantum Spin-1 Chains, Phys. Rev. Lett. 109, 207202 (2012).

[88] Z. Zhang, A. Ahmadain, and I. Klich, Novel quantum phase transition from bounded to extensive entanglement, Proc. Natl. Acad. Sci. USA 114, 5142 (2017).

[89] R. Movassagh and P. W. Shor, Supercritical entanglement in local systems: Counterexample to the area law for quantum matter, Proc. Natl. Acad. Sci. USA 113, 13278 (2016).

[90] L. Levine and R. Movassagh, The gap of the area-weighted Motzkin spin chain is exponentially small, J. Phys. A: Math. Theor. 50, 255302 (2017).
[91] R. Movassagh, Entanglement and correlation functions of the quantum Motzkin spin-chain, J. Math. Phys. (Melville, NY) 58, 031901 (2017)

[92] L. Dell'Anna, O. Salberger, L. Babiero, A. Trombettoni, and V. E. Korepin, Violation of cluster decomposition and absence of light cones in local integer and half-integer spin chains, Phys. Rev. B 94, 155140 (2016).

[93] L. Babiero, L. Dell'Anna, A. Trombettoni, and V. E. Korepin, Haldane topological orders in Motzkin spin chains, Phys. Rev. B 96, 180404(R) (2017).

[94] X. Chen, E. Fradkin, and W. Witczak-Krempa, Gapless quantum spin chains: Multiple dynamics and conformal wavefunctions, J. Phys. A: Math. Theor. 50, 464002 (2017).

[95] X. Chen, E. Fradkin, and W. Witczak-Krempa, Quantum spin chains with multiple dynamics, Phys. Rev. B 96, 180402(R) (2017).

[96] F. Sugino and P. Padmanabhan, Area law violations and quantum phase transitions in modified Motzkin walk spin chains, J. Stat. Mech.: Theory Exp. (2018) 013101.

[97] M. Medenjak, K. Klobas, and T. Prosen, Diffusion in Deterministic Interacting Lattice Systems, Phys. Rev. Lett. 119, 110603 (2017).

[98] S. Gopalakrishnan and B. Zakirov, Facilitated quantum cellular automata as simple models with non-thermal eigenstates and dynamics, Quantum Sci. Technol. 3, 044004 (2018).

[99] Generally, the dimension of a Krylov space for a chain of $L$ sites can be derived combinatorially and is given by $\mathcal{D}_{d u}=$ $\sum_{i \geqslant 0}^{2 i+N_{d u} \leqslant L} \frac{N_{d u}+1}{i+N_{d u}+1}\left(\begin{array}{c}L \\ 2 i+N_{d u}\end{array}\right)\left(\begin{array}{c}2 i+N_{d u} \\ i\end{array}\right)$, which only depends on the sum $N_{d u}=N_{d}+N_{u}$ of unpaired up and down spins [87].

[100] See Supplemental Material at http://link.aps.org/ supplemental/10.1103/PhysRevResearch.4.L012003 for details on Krylov-space restricted thermalization, the construction of $\left|\mathcal{S}_{v}\right\rangle$, dynamical quantum typicality, cellular automaton dynamics, additional data on domain-wall melting, entanglement growth, and quench dynamics, as well as the derivation of the spin-current operator, including Refs. [101-106].

[101] V. Oganesyan and D. A. Huse, Localization of interacting fermions at high temperature, Phys. Rev. B 75, 155111 (2007).

[102] H. Liu and S. J. Suh, Entanglement Tsunami: Universal Scaling in Holographic Thermalization, Phys. Rev. Lett. 112, 011601 (2014).

[103] C. Chiaracane, F. Pietracaprina, A. Purkayastha, and J. Goold, Quantum dynamics in the interacting Fibonacci chain, Phys. Rev. B 103, 184205 (2021).

[104] H. Fehske, J. Schleede, G. Schubert, G. Wellein, V. S. Filinov and A. R. Bishop, Numerical approaches to time evolution of complex quantum systems, Phys. Lett. A 373, 2182 (2009).

[105] M. Žnidarič, Entanglement growth in diffusive systems, Commun. Phys. 3, 100 (2020).

[106] T. Rakovszky, F. Pollmann, and C. von Keyserlingk, Entanglement growth in diffusive systems with large spin, Commun. Phys. 4, 91 (2021).

[107] D. S. Rokhsar and S. A. Kivelson, Superconductivity and the Quantum Hard-Core Dimer Gas, Phys. Rev. Lett. 61, 2376 (1988).

[108] With the exception of Fig. 4, we choose $c_{1}=c_{3}=1$ and $c_{2}=$ -1 throughout this Letter, for which the $\left|\mathcal{S}_{v}\right\rangle$ are located close to the center of the spectrum. 
[109] Note that the specific site $\ell$ is irrelevant in the case of PBCs, while for OBCs, we use $\ell=L / 2$.

[110] J. Richter, N. Casper, W. Brenig, and R. Steinigeweg, Magnetization dynamics in clean and disordered spin-1 XXZ chains, Phys. Rev. B 100, 144423 (2019).

[111] F. Jin, D. Willsch, M. Willsch, H. Lagemann, K. Michielsen, and H. De Raedt, Random state technology, J. Phys. Soc. Jpn. 90, 012001 (2021).

[112] T. Heitmann, J. Richter, D. Schubert, and R. Steinigeweg, Selected applications of typicality to real-time dynamics of quantum many-body systems, Z. Naturforsch. A: Phys. Sci. 75, 421 (2020).

[113] H. Singh, B. A. Ware, R. Vasseur, and A. J. Friedman, Subdiffusion and Many-Body Quantum Chaos with Kinetic Constraints, Phys. Rev. Lett. 127, 230602 (2021).

[114] K. Adhikari and K. S. D. Beach, Slow dynamics of the Fredkin spin chain, Phys. Rev. B 104, 115149 (2021).

[115] C. L. Henley, From classical to quantum dynamics at RokhsarKivelson points, J. Phys.: Condens. Matter 16, S891 (2004).

[116] C. Castelnovo, C. Chamon, C. Mudry, and P. Pujol, From quantum mechanics to classical statistical physics: Generalized Rokhsar-Kivelson Hamiltonians and the "Stochastic Matrix Form" decomposition, Ann. Phys. (Amsterdam) 318, 316 (2005).

[117] B. Tong, O. Salberger, K. Hao, and V. Korepin, ShorMovassagh chain leads to unusual integrable model, J. Phys. A: Math. Theor. 54, 394002 (2021).

[118] T. Rakovszky, F. Pollmann, and C. W. von Keyserlingk, Sub-ballistic Growth of Rényi Entropies due to Diffusion, Phys. Rev. Lett. 122, 250602 (2019).

[119] Y. Huang, Dynamics of Rényi entanglement entropy in diffusive qudit systems, IOP SciNotes 1, 035205 (2020).
[120] D. Gobert, C. Kollath, U. Schollwöck, and G. Schütz, Realtime dynamics in spin- $\frac{1}{2}$ chains with adaptive time-dependent density matrix renormalization group, Phys. Rev. E 71, 036102 (2005).

[121] J. Hauschild, F. Heidrich-Meisner, and F. Pollmann, Domainwall melting as a probe of many-body localization, Phys. Rev. B 94, 161109(R) (2016).

[122] M. Ljubotina, M. Žnidarić, and T. Prosen, Spin diffusion from an inhomogeneous quench in an integrable system, Nat. Commun. 8, 16117 (2017).

[123] M. Medenjak and J. De Nardis, Domain wall melting in spin-1 XXZ chains, Phys. Rev. B 101, 081411(R) (2020).

[124] For such cases, with one macroscopically populated eigenstate, a typicality-based framework allows us to predict the relaxation of observables based on $\mathcal{L}(t)[100,125]$.

[125] P. Reimann, B. N. Balz, J. Richter, and R. Steinigeweg, Temporal relaxation of gapped many-body quantum systems, Phys. Rev. B 101, 094302 (2020).

[126] We refer to the Rényi entropies $S_{\alpha}(t)=\ln \operatorname{Tr}\left[\rho_{A}^{\alpha}\right] /(1-\alpha)$, which yield $S_{1}(t)=-\operatorname{Tr}\left[\rho_{A} \ln \rho_{A}\right]$ for $\alpha=1$.

[127] D. K. Mark, C.-J. Lin, and O. I. Motrunich, Unified structure for exact towers of scar states in the Affleck-Kennedy-LiebTasaki and other models, Phys. Rev. B 101, 195131 (2020).

[128] S. Moudgalya, N. Regnault, and B. A. Bernevig, $\eta$-pairing in Hubbard models: From spectrum generating algebras to quantum many-body scars, Phys. Rev. B 102, 085140 (2020).

[129] K. Pakrouski, P. N. Pallegar, F. K. Popov, and I. R. Klebanov, Many-Body Scars as a Group Invariant Sector of Hilbert Space, Phys. Rev. Lett. 125, 230602 (2020).

[130] N. O’Dea, F. Burnell, A. Chandran, and V. Khemani, From tunnels to towers: Quantum scars from Lie algebras and $q$ deformed Lie algebras, Phys. Rev. Research 2, 043305 (2020). 